\title{
Cathepsin $\mathrm{V}$ is involved in the degradation of invariant chain in human thymus and is overexpressed in myasthenia gravis
}

\author{
Eva Tolosa, ${ }^{1}$ Weijie Li, ${ }^{2}$ Yoshiyuki Yasuda, ${ }^{2}$ Wolfgang Wienhold, ${ }^{1}$ Lisa K. Denzin, ${ }^{3}$ \\ Alfred Lautwein, ${ }^{4}$ Christoph Driessen, ${ }^{4}$ Petra Schnorrer, ${ }^{1}$ Ekkehard Weber, ${ }^{5}$ \\ Stefan Stevanovic, ${ }^{6}$ Raffael Kurek, ${ }^{7}$ Arthur Melms, ${ }^{1}$ and Dieter Brömme ${ }^{2}$ \\ ${ }^{1}$ Department of Neurology, Tübingen University Hospital, Tübingen University, Tübingen, Germany \\ ${ }^{2}$ Department of Human Genetics, Mount Sinai School of Medicine, New York, New York, USA \\ ${ }^{3}$ Memorial Sloan-Kettering Cancer Center, New York, New York, USA \\ ${ }^{4}$ Department of Medicine, Tübingen University Hospital, Tübingen University, Tübingen, Germany \\ ${ }_{5}^{5}$ Institute of Physiological Chemistry, Martin-Luther-University Halle-Wittenberg, Department of Medicine, \\ Halle, Germany \\ ${ }^{6}$ Department of Immunology, Institute for Cell Biology, Tübingen University Hospital, Tübingen University, \\ Tübingen, Germany \\ ${ }^{7}$ Department of Gynecology and Obstetrics, Tübingen University Hospital, Tübingen University, Tübingen, Germany
}

Stepwise degradation of the invariant chain (Ii) is required for the binding of antigenic peptides to MHC class II molecules. Cathepsin (Cat) L in the murine thymus and Cat S in peripheral APCs have both been implicated in the last step of Ii degradation that gives rise to the class II-associated invariant chain peptides (CLIP). Cat $V$ has been recently described as highly homologous to Cat $\mathrm{L}$ and exclusively expressed in human thymus and testis, but with no mouse orthologue. We report that $\mathrm{Cat} \mathrm{V}$ is the dominant cysteine protease in cortical human thymic epithelial cells, while Cat $\mathrm{L}$ and Cat $\mathrm{S}$ seem to be restricted to dendritic and macrophage-like cells. Active Cat V in thymic lysosomal preparations was demonstrated by active-site labeling. Recombinant $C a t V$ was capable of converting Ii into CLIP efficiently, suggesting that Cat V is the protease that controls the generation of $\alpha \beta$-CLIP complexes in the human thymus, in analogy to Cat $\mathrm{L}$ in mouse. Comparison of Cat $\mathrm{V}$ expression between thymi from patients with myasthenia gravis and healthy controls revealed a significantly higher expression level in the pathological samples, suggesting a potential involvement of this protease in the immunopathogenesis of myasthenia gravis, an autoimmune disease almost invariably associated with thymic pathology.

J. Clin. Invest. 112:517-526 (2003). doi:10.1172/JCI200318028.

\section{Introduction}

Antigens presented by APCs are either recognized by CD8 T cells or CD4 T cells. CD8 T cells specifically recognize antigenic peptides from cytoplasmic antigens that have been processed in the cytosol by the proteasomal degradation pathway and are bound to

Received for publication February 4, 2003, and accepted in revised form May 13, 2003.

Address correspondence to: Eva Tolosa, Neuroimmunology, Department of Neurology, Tübingen University Hospital, Auf der Morgenstelle 15, 72076 Tübingen, Germany. Phone: 49-7071-2987638; Fax: 49-7071-295201;

E-mail: eva.tolosa@uni-tuebingen.de.

Eva Tolosa and Weijie Li contributed equally to this work. Arthur Melms and Dieter Brömme share senior authorship. Conflict of interest: The authors have declared that no conflict of interest exists.

Nonstandard abbreviations used: cathepsin (Cat); class II-associated invariant chain peptides (CLIP); leucylhomophenylalanine-vinylsulfone (LHVS); human leukocyte antigen (HLA); invariant chain (Ii); thymic epithelial cell (TEC); myasthenia gravis (MG); laser capture microdissection (LCM); $\alpha$ and $\beta$ class II molecule invariant chain $(\alpha \beta \mathrm{Ii})$; major outer membrane protein (MOMP).
MHC class I molecules. CD4 T cells detect extracellular pathogens or self-proteins that have been processed in the endocytic compartment and that are presented on MHC class II molecules. MHC class II antigen presentation requires at least two distinct proteolytic events: (1) intravesicular degradation of the antigen, and (2) processing of the MHC-bound invariant chain (Ii) into class II-associated invariant chain peptides (CLIP). Lysosomal cysteine proteases have been implicated in both processes $(1,2)$. For example, asparaginyl endopeptidase, a human cysteine protease related to the plant enzyme legumain, has been shown to generate a naturally processed $T$ cell epitope of tetanus toxin (3). Cathepsins (Cats) B, S, and L of the papain superfamily are most likely involved in the degradation of the bulk lysosomal protein load and may contribute to the generation of antigenic peptides (4). Before the binding of antigenic peptides, the MHC class II complex has to be freed from the Ii that blocks the binding groove for the peptide $(5,6)$. The degradation of Ii, and hence peptide loading, can be blocked by Cat inhibitors such as leupeptin or leucylhomophenylalanine-vinylsulfone (LHVS) $(7,8)$. Both 
inhibitors prevent the generation of CLIP, a set of 3 -kDa peptides that will be replaced by antigenic peptides in the binding groove of the MHC complex in a reaction catalyzed by the human leukocyte antigen-DM (HLA-DM) molecule (9). The generation of CLIP in B cells and dendritic cells is mediated by Cat $\mathrm{S}$, a papain-like cysteine protease predominantly expressed in lymphatic tissues and spleen (10). However, experiments with $\mathrm{KO}$ mice demonstrated that Cat $\mathrm{S}$ is not universally responsible for generation of CLIP in all types of APCs. For example, antigen presentation in the absence of Cat $S$ in macrophages was less affected than in B cells (11), indicating either Ii-processing Cat activities are redundant in APCs or distinct APCs express cell/tissue-specific cathepsins. Indeed, Shi et al. (12) could show that in Cat L- and $\mathrm{S}$-deficient macrophages, Cat $\mathrm{F}$ can efficiently process the Ii. On the other hand, Nakagawa et al. (13) demonstrated that Cat L-deficient mice exhibit an impairment of thymic antigen presentation resulting in low numbers of $\mathrm{CD}^{+}$cells, and suggested that Cat $\mathrm{L}$ activity is responsible for the final degradation of $\mathrm{Ii}$ in cortical thymic epithelial cells (TECs).

We have recently identified and partially characterized a novel human cysteine protease closely related to Cat $\mathrm{L}$ that we named Cat V. Cat V shares $80 \%$ protein sequence identity with Cat $\mathrm{L}$, but in contrast to the ubiquitously expressed Cat $\mathrm{L}$, its expression is restricted to thymus and testis (14-16). Despite significant efforts to identify a mouse orthologue of human Cat $V$ with a thymus-specific expression pattern, this protease has not been found. Interestingly, at the protein level, mouse Cat $\mathrm{L}$ has a higher sequence homology to human Cat $\mathrm{V}$ than to human Cat L (14). The high degree of sequence identity between both human cathepsins and their identical location on the long arm of human chromosome 9 (14) indicate a more recent gene duplication event that might date back approximately 100 Mio years, to a time when the major mammalian radiation occurred. Thus, it appears possible that mouse Cat $\mathrm{L}$ represents the ancestor for both human Cat $\mathrm{V}$ and $\mathrm{L}$. Considering the thymus-specific expression of Cat $\mathrm{V}$, we asked whether Cat $\mathrm{V}$ might play a role in antigen presentation in the human thymus that has been proposed for mouse Cat $\mathrm{L}$ in the mouse thymus. In this report, we describe the specific expression and localization of Cat $\mathrm{V}$ in normal human thymus, its overexpression in the thymus of patients with myasthenia gravis (MG), and the degradation of Ii by the protease.

\section{Methods}

Patients and thymic tissue sample processing. Normal human thymi were obtained according to Institutional Review Board guidelines from 2-day-old to 14year-old donors undergoing corrective cardiac surgery. Thymi from patients with MG were obtained during therapeutic thymectomy. Representative blocks of each tissue sample were frozen and stored at $-70^{\circ} \mathrm{C}$ for RNA extraction. The remaining tissue was cut into pieces and digested for 45 minutes at $37^{\circ} \mathrm{C}$ with collagenase $A(1 \mathrm{mg} / \mathrm{ml}$, Boehringer, Mannheim, Germany) and deoxyribonuclease $(5 \mu \mathrm{g} / \mathrm{ml}$, SigmaAldrich, St. Louis, Missouri, USA) in RPMI 1640. After digestion, several rounds of 1 -g sedimentation eliminated a great number of thymocytes. The pellets, an epithelial-enriched cell fraction, were plated on collagen-coated (BD Biosciences, Bedford, Massachusetts, USA) plates in Optimem (Gibco, Paisley, United Kingdom) a low-serum-containing medium that prevented the outgrowth of fibroblasts. After 7 to 10 days of culture, a monolayer consisting of more than $95 \%$ TECs was obtained.

Preparation of thymic lysosomal extracts. Lysosomal fractions were prepared from the freshly digested epithelial-enriched cell fraction (pellets after sedimentation rounds) by differential centrifugation, followed by hypotonic lysis as previously described (17).

Laser capture microdissection of thymic tissue. Freshly cut thymic sections of less than $10 \mu \mathrm{m}$ were fixed in $70 \%$ ethanol and stained with Meyer's hematoxylin and eosin. Slides were dehydrated and, after a final xylene incubation, were air-dried for at least 10 minutes. The stained slides were immediately used for laser capture microdissection (LCM). Microdissection of thymic cortex and medulla from the sections of human thymi was performed with the use of an Arcturus PixCell II Laser Capture microdissection microscope (Arcturus Engineering Inc., Mountain View, California, USA). Medullary and cortical portions of the thymus section were independently collected on a thermoplastic polymer film-coated cap. Special attention was dedicated to avoid cross-contamination of medullary and cortical cells and to avoid the dendritic cell-rich corticomedullary areas. RNA was extracted immediately from the transfer film-attached cells.

Real-time quantitative PCR. Cells and tissue were lysed in Trizol (Invitrogen, Grand Island, New York, USA), and the RNA extracted according to the manufacturer's protocol. Glycogen (Roche Diagnostics, Mannheim, Germany) was added as a carrier to the LCM samples. RNA was dissolved in water and stored at $-70^{\circ} \mathrm{C}$. First-strand cDNA was prepared from total RNA by reverse transcription using the Superscript RNase $\mathrm{H}^{-}$Reverse Transcriptase (Invitrogen) and random hexamers. Gene expression was measured in the ABI Prism 7700 Sequence Detection System (Applied Biosystems, Foster City, California, USA). Primers (B\&G Biotech, Freiburg, Germany) were selected with the Husar Genius software (DKFZ, Heidelberg, Germany) to span exon-intron junctions to prevent amplification of genomic DNA and to result in amplicons less than $150 \mathrm{bp}$ to enhance efficiency of PCR amplification. The sequences of the primers are as follows: Cat V, forward 5'-TGGAAGGCAACACACAGAAG-3', reverse 5'-GAAGCCATGTTTCCCTTGG-3'; Cat L, forward 5'-ACCAAGTGGAAGGCGATG-3', reverse 5'TTCCCTTCCCTGTATTCCTG-3'; Cat S, forward 5'- 
ACTCAGAATGTGAATCATGGTG-3', reverse 5' -TTCTTGCCATCCGAATATATCC-3'. As endogenous control, primers specific for human $18 \mathrm{~S}$ ribosomal RNA were used, forward 5'-CGGCTACCACATCCAAGGAA-3' and reverse $5^{\prime}$-GCTGGAATTACCGCGGCT-3'. PCR products were directly cloned into the vector PCR 2.1-TOPO by using the TOPO TA Cloning Kit (Invitrogen, Groningen, The Netherlands) and the cloned products were sequenced to confirm primer specificity by using the AmpliTaq FS BigDye deoxy terminator cycle sequencing kit and read in a PE-ABI Prism 310 automated Sequencer (Applied Biosystems). Product specificity of each PCR product was further confirmed by agarose gel electrophoresis and/or by dissociation curve analysis. Amplification of specimens and serial dilutions of the amplicon-standards was carried out in a total volume of $15 \mu$ l Sybrgreen Master Mix (Applied Biosystems) and primers at optimized concentrations. Standard curves were generated for each gene and the amplification was found to be $90 \%$ to $100 \%$ efficient, as determined by the slopes of the standard curves. Standard curve extrapolation of copy number was calculated for the gene of interest to have a fair approximation to the number of copies present in each sample. Relative quantitation of gene expression was determined with the use of the comparative $\mathrm{C}_{\mathrm{T}}$ method as suggested by the manufacturers. All results are normalized with respect to the internal control 18S rRNA, and are expressed relative to the levels found in one of the samples.

Recombinant buman cathepsins. Human Cat V was expressed in Pichia pastoris by using the expression vector pPic-9 (Invitrogen, Carlsbad. California, USA), as previously described (14). Human Cat L was expressed using the same expression system (unpublished results, D. Brömme). Human Cat S was expressed in $S f 9$ cells using the baculovirus expression system (18). Cats V, L, and $S$ were activated by treatment with pepsin and purified on SP-Sepharose (Pharmacia, Uppsala, Sweden) as previously reported $(14,18)$. The active site concentrations of the purified proteases were determined by E-64 titration (19).

Immunobistochemistry and antibodies. Monoclonal antihuman Cat $\mathrm{L}$ antibodies $(\mathrm{mAb})$ were generated by immunization of $\mathrm{A} / \mathrm{J}$ mice with human pro-Cat $\mathrm{L}$ purified from the cell culture medium of human nonsmall cell lung cancer cell line EPLC $32 \mathrm{M} 1$, as previously described (20). More than 400 hybridoma clones were screened by ELISA or Western blot analysis and 50 clones were found to secrete antibodies that reacted with Cat $\mathrm{L}$ or its precursor. We screened four epitope-characterized antibodies for their ability to discriminate between human Cats $\mathrm{L}$ and $\mathrm{V}$ and selected mAb33/1 (specific for Cat L) and mAb33/2 (Cat $\mathrm{L}$ and Cat V) for this study. Western blot analysis of recombinant human Cats $L$ and $V$ were used to determine the specificities of the antibodies. For the staining of Cat $S$, a rabbit polyclonal antiserum was used as previously described (21).
Paraffin sections $(5 \mu \mathrm{m})$ were mounted onto Vectabond slides (Vector Laboratories, Burlingame, California, USA) dried overnight, and dewaxed with xylene. Sections were incubated with mouse mAbs antihuman Cat $\mathrm{L} 33 / 1$ and 33/2, and with rabbit serum against Cat $S$, followed by incubation with biotinylated goat antimouse or antirabbit secondary antibodies (Biogenix, San Ramon, California, USA). Peroxidase-labeled avidin was used to localize the secondary antibody with oxidized diaminobenzidine as chromogen (Biogenix). Counterstaining was performed with Meyer's hematoxylin and eosin. All slides were evaluated using a Nikon Eclipse E800 (Enfield, Connecticut, USA) or a Zeiss Axioplan microscope (Carl Zeiss Deutschland, Jena, Germany).

Western blot analysis. Protein samples were subjected to $10 \%$ SDS-polyacrylamide electrophoresis and transferred onto nitrocellulose membranes. The mAb33/1, mAb33/2 (1: 5000 dilution), or mAb 2362D8 (kind gift from I. Santamaria, University Oviedo, Spain) were used for analyses. After incubation with HRP-conjugated goat antimouse secondary antibody, HRP activity was detected with the use of the LumiLight Substrate (Boehringer Mannheim, Mannheim, Germany) and visualized by chemiluminescence.

Active site labeling of cysteine proteinases. Active site labeling experiments of cysteine proteinases were performed as previously described (22) with some modifications. Briefly, lysosomal fractions from human thymus tissues were incubated with or without LHVS, a highly potent irreversible Cat $S$ inhibitor (10) (kindly provided by Celera Corp., South San Francisco, California, USA) $(1 \mathrm{nM}$ or $1 \mu \mathrm{M})$ at $37^{\circ} \mathrm{C}$ for 20 minutes before incubation with biotin-conjugated DCG-04 $(50 \mu \mathrm{M})$ for 1 hour at $37^{\circ} \mathrm{C}$. Recombinant human Cats $V$ and $S$ (10 ng each) were incubated with $1 \mathrm{nM} \mathrm{LHVS}$ at $37^{\circ} \mathrm{C}$ for increasing periods $(0$, $1,5,20$, and 60 minutes) before labeling with DCG04 (kindly provided by M. Bogyo, Celera Corp.). The labeling reaction was stopped by the addition of SDS sample buffer. The samples were separated by $10 \%$ SDS-PAGE and transferred on PVDF membranes. After blocking with $1 \%$ BSA/T-PBS, membranes were probed with streptavidin-HRP at room temperature for 1 hour. DCG-04 inhibited proteases were visualized with the use of the ECL system.

In vitro digestion of invariant chain complexes. HLA-DR3 $\alpha$ and $\beta$ class II molecule invariant chain $(\alpha \beta \mathrm{Ii})$ complexes were purified with the use of a DA6.147 affinity column from a Pala B-lymphoblastoid cell line extract that was labeled with $\left[{ }^{35} \mathrm{~S}\right]$ methionine for 4 hours in the presence of $10 \mu \mathrm{M}$ monensin (SigmaAldrich) as previously described (23). Purified, radiolabeled $\alpha \beta$ Ii complexes $(50,000 \mathrm{cpm})$ were treated with Cats $\mathrm{V}, \mathrm{L}$, or $\mathrm{S}$ in $0.1 \mathrm{M}$ sodium acetate, $\mathrm{pH} 5.0$ containing 3 mM DTT, 3 mM EDTA, $12 \mu \mathrm{M}$ Pepstatin $\mathrm{A}$, and $0.1 \% \mathrm{C}_{12} \mathrm{E}_{9}$ (Sigma-Aldrich) for 2 hours at $37^{\circ} \mathrm{C}$. After treatment, samples were neutralized with the addition of $1 \mu \mathrm{l}$ of $1 \mathrm{M}$ Tris, mixed with $10 \times$ 
concentrated nonreducing Laemmli sample buffer and separated by SDS-PAGE ( $15 \%$ acrylamide). As controls, enzymes were treated with the protease inhibitor, E-64 $(50 \mu \mathrm{M})$, before the addition of radiolabeled $\alpha \beta$ Ii complexes.

In vitro loading of Cat-digested $\alpha \beta$ Ii complexes by HLA-DM. Affinity-purified, radiolabeled DR3- $\alpha \beta$ Ii complexes were digested with Cats V $(50 \mathrm{nM}), \mathrm{L}(10 \mathrm{nM})$, or S (50 $\mathrm{nM})$, as described previously, and $50 \mu \mathrm{M}$ E-64 was added to each sample to inactivate the cathepsins (10 minutes, room temperature). The DR3-specific major outer membrane protein (MOMP) peptide $(1 \mu \mathrm{M}$, QASLALSYRLNMFTP) (24) was added to the samples in the presence and absence of HLA-DM (9) that had been affinity purified from T2/DM cells (25). The $\mathrm{pH}$ of the reaction mixture was adjusted to 5.0 by the addition of $1 \mathrm{M}$ acetic acid, and the samples were incubated for 2 hours at $37^{\circ} \mathrm{C}$. Samples were neutralized with $1 \mathrm{M}$ Tris, mixed with $10 \times$ concentrated nonreducing Laemmli sample buffer and separated by SDS-PAGE (11.25\%).

\section{Results}

$m R N A$ expression of Cats $V, L$, and $S$ in thymus. Cats $S$ and $L$ have been implicated in the degradation of murine invariant chain and may play a crucial role in antigen presentation in peripheral APCs and the thymus, respectively. By means of quantitative real-time PCR, we have analyzed the mRNA expression profile of Cats $\mathrm{S}, \mathrm{L}$, and $\mathrm{V}$ in normal human thymus and compared it with PBMCs. Cat $\mathrm{S}$ was found in greater amounts in PBMCs than in thymus (in average 50 times more, Figure 1a), and within the thymus, it was undetectable by real-time PCR in purified epithelial cells and in thymocytes (data not shown), suggesting that the expressing cells are thymic dendritic cells and macrophages. Consistent with earlier data from the murine model (13), Cat L was found expressed both in the thymus and in PBMCs at similar levels, but at much lower levels than Cat $S$ in PBMCs. Cat V was found exclusively in the thymus and was undetectable in PBMCs. Of note, the levels of Cat $S$ in PBMCs were similar to the levels of Cat $\mathrm{V}$ in thymus, whereas Cat $\mathrm{L}$, although clearly detectable in both samples, was expressed at much lower levels. To study the distribution of this protease in human thymus, normal tissue was enzymatically digested and thymocytes were isolated by ficoll centrifugation. An epithelial cell-enriched fraction was obtained after sequential sedimentation of freshly digested tissue that eliminated a high proportion of thymocytes. Cat

\section{Figure 1}

$V$ was found exclusively in this epithelial cell-enriched fraction, whereas purified thymocytes were completely devoid of it (Figure 1b). This finding was confirmed in primary cultures of thymic epithelium with a $95 \%$ content of cytokeratin-positive cells (not shown). To determine where the transcription of Cat $\mathrm{V}$ occurs in the thymus, fragments of medulla and cortex were isolated from tissue sections by LCM. Real-time quantitative PCR of five microdissected cortex-medulla sample sets revealed that Cat $\mathrm{V}$ mRNA is expressed at 12-fold higher levels in the thymic cortex compared with the medulla (Figure 1, $c$ and d).

Characterization of antibuman Cat $L$ antibodies. Two mAbs recognizing either epitope GYGFEST ( $\mathrm{mAb33} / 2$ ) or epitope FYKE (mAb33/1) in Cat L were thus tested for cross reactivities with human Cat V. Whereas $\mathrm{mAb33} / 2$ recognized both cathepsins, $\mathrm{mAb} 33 / 1$ revealed no cross reactivity with Cat $\mathrm{V}$ in Western blot analysis with the use of recombinant human Cats L and V, respectively (Figure 2a). Both antibodies did not show cross reactivities to other human cathepsins (recombinant Cats B, K, S, F, and $\mathrm{W}$, not shown). Immunoadsorption of both antibodies with nitrocellulose membrane-immobilized recombinant $C a t \mathrm{~L}$ prevented the detection of immune-positive material by Western blot analysis, whereas preincubation of nitrocellullose blots containing Cat $\mathrm{V}$ protein only abolished the activity of $\mathrm{mAb33} / 2$ (not shown). Hence, $\mathrm{mAb33} / 2$ and mAb33/ 1 could be used to discriminate between the protein expression of Cats $\mathrm{V}$ and $\mathrm{L}$ in thymus. To characterize the tissue immunostaining efficacy of both mAbs, we chose spleen specimens known
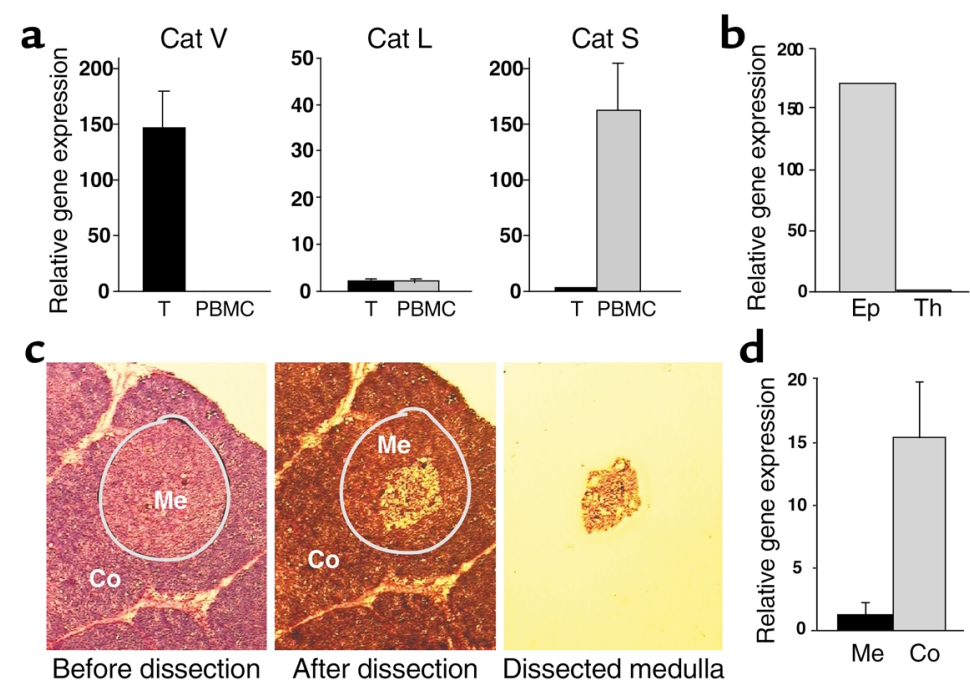

Before dissection

mRNA expression of Cats V, L, and S. (a) Real-time RT-PCR analysis for the expression of Cats V, L, and S in thymus ( $T$, black bars) and PBMCs (gray bars). The graphs display the averaged values of seven thymi and five PBMC samples. (b) Cat $V$ in the thymus is exclusively found in an epithelial cell-enriched fraction (Ep) and could not be detected in thymocytes (Th). (c and d) Laser capture microdissection of the thymic medulla (Me, black bar) and cortex (Co, gray bar), followed by RT-PCR analysis shows that Cat $\mathrm{V}$ is mostly found in the cortex of the thymus. 


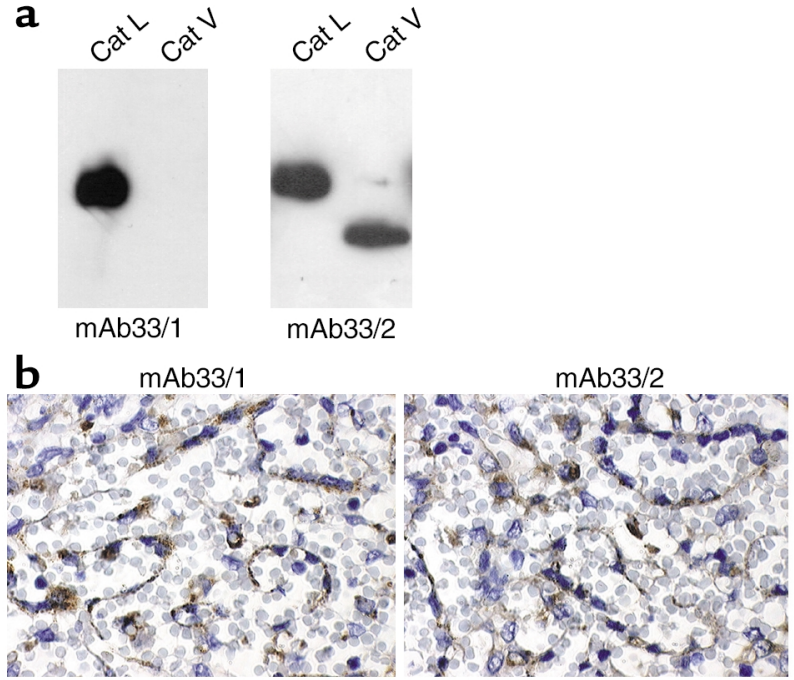

to express Cat L but not Cat V (14). Both antibodies stained the epithelial cells with comparable intensity (Figure 2b).

Immunostaining of Cats $V$ and $L$ in normal thymus. Paraffin-embedded thymus specimens from three normal donors analyzed with mAb33/ 1 revealed only a sparse staining of the thymic cortex limited to single macrophages (Figure 3, a, c, and e). No staining was observed in cortical epithelial cells suggesting that the thymic cortex does not express Cat L outside macrophages (Figure 3, a and c). Within the medulla, a larger number of macrophages expressed Cat $\mathrm{L}$ (Figure 3, a and c), whereas the epithelial component remained again unstained. It should be noted that most macrophages are vacuolated, indicating that they are actively involved in the engulfment of apoptotic thymocytes. In contrast, $\mathrm{mAb} 33 / 2$, which cross reacts with Cats $V$ and $L$, revealed a strong staining of the cortex (Figures 3, b and d, and 6a). The staining pattern was clearly indicative for the expression of Cat V in the cortical epithelial cell framework (Figures $3 \mathrm{~d}$ and $6 \mathrm{a}$ ). Cat $\mathrm{V}$ positive cells surrounded Cat $\mathrm{V}$ negative lymphocytes throughout the cortex. Staining of medullary epithelial cells was significantly weaker when compared with their counterparts in the cortex (not shown). The staining of macrophages within the medulla is comparable with that of the staining pattern of $\mathrm{mAb33} / 1$, indicating that $\mathrm{mAb33} / 2$ recognizes Cat $\mathrm{L}$ in these cells (Figure 3 , $a$ and $b$ ). Because of the cross reactivity of $\mathrm{mAb33} / 2$ with Cats $\mathrm{L}$ and $\mathrm{V}$, we cannot entirely exclude the expression of $\mathrm{Cat} \mathrm{V}$ in macrophages, although the absence or low levels of Cat V mRNA in peripheral monocytes and thymic medulla, respectively, make this unlikely. Cat $\mathrm{S}$ expression was more prominent in the corticomedullary junction, where dendritic cells accumulate, but epithelial cells were negative in both compartments. None of the cathepsins revealed a significant expression in thymocytes (Figure 3, e and f). Entirely consistent with both our

\section{Figure 2}

Specificity of mAb33/ 1 and $m A b 33 / 2$ for human Cats $V$ and L. (a) Western blot analysis of recombinant human Cats $V$ and $L$ using $\mathrm{mAb33} / 1$ and $\mathrm{mAb33} / 2$. Both enzymes, $0.05 \mu \mathrm{g}$, were loaded onto $12 \%$ SDS Tris-glycine gels and antibody dilutions of 1:5000 were used. The $\mathrm{mAb} 33 / 2$ cross reacts with both proteases, whereas mAb33/1 only recognizes Cat $L$. (b) The mAb33/1 and mAb33/2 recognize $C a t L$ in human spleen tissue sections and are indistinguishable in their sensitivity toward Cat $L$ antigen in paraffinembedded tissue section. The ABC staining kit, Super Sensitive Ready-To-Use Detection System (Biogenex), was used for immunohistochemical protein localization.

immunohistochemical data and the results at the transcriptional level, Western blot analysis that used a specific antibody against Cat $\mathrm{V}$ revealed the presence of this protease in both whole thymic tissue and in a freshly isolated fraction enriched for epithelial cells, whereas Cat $\mathrm{V}$ was absent from purified thymocytes (Figure 4).
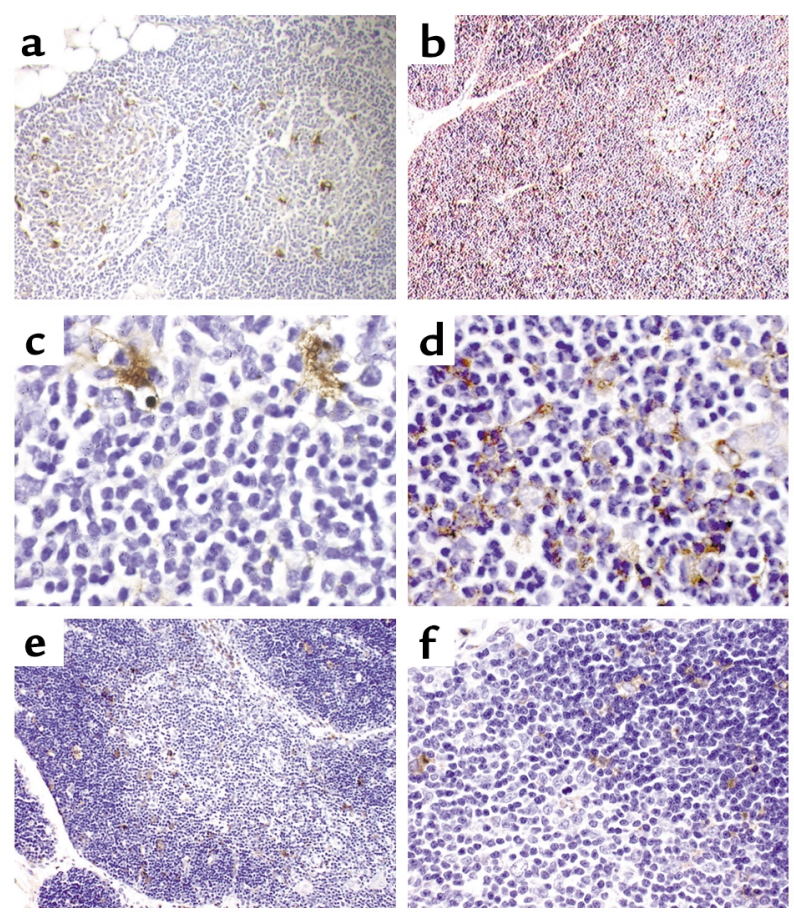

Figure 3

Immunohistochemical analysis of Cats $\mathrm{V}, \mathrm{L}$, and $\mathrm{S}$ expression in thymus. Mouse monoclonal antihuman $C a t ~ L$ antibodies mAb33/1 (a and c; recognizes only Cat L), mAb33/2 (b and d; cross reacts with human Cats $\mathrm{V}$ and $\mathrm{L}$ ) and a rabbit polyclonal antibody against $C$ at $S$ (e and $\mathbf{f}$ ) were used to localize these proteases in human thymus. $\mathbf{a}$ and $\mathbf{c}$ show the expression of $C$ at $L$ in macrophage-like cells of the normal thymus. $\mathbf{b}$ shows the expression of $C a t ~ V$ in thymic cortex. Immunopositive cells in the medulla may express either Cats $L$ and $V$ or only Cat $L$. $\mathbf{d}$ demonstrates the expression in cortical thymic epithelial cells, which do not express Cat $L$ (see $\mathbf{c}$ ). e and $\mathbf{f}$ show the discrete staining for $C$ at $S$ both in the cortex and medulla with a rabbit polyclonal antibody specific for Cat $S$. All magnifications are $\times 1000$ with the exception of $\mathbf{a}$ and $\mathbf{e}(\times 200)$ and $\mathbf{b}(\times 400)$. Tissue sections were counterstained with hematoxylin. 


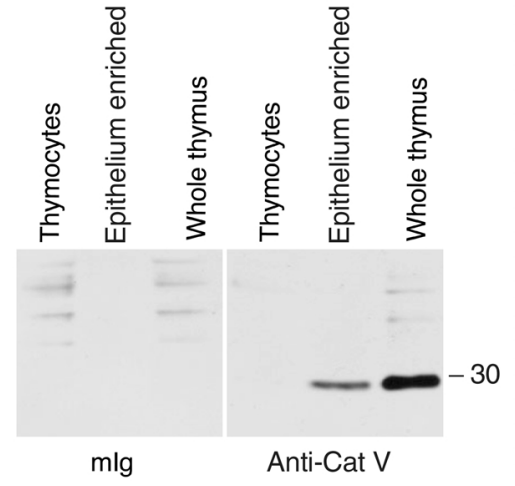

Figure 4

Western blot. Purified thymocytes, an epithelium-enriched fraction of human thymus and whole thymus, were analyzed by Western blot with anti-Cat $\mathrm{V}$-specific mAb 236.2D8 (right panel) and control antibody mouse $\mathrm{lg}$ ( $\mathrm{mlg}$ ) (left panel). A strong Cat $\mathrm{V}$ immunoreactive band below the $30-\mathrm{kDa}$ marker is seen in the epitheliumenriched and whole thymus fraction, but not in the thymocytes.

Cat $V$ is expressed as mature and active protease in the thymus. Lysosomal preparations of human thymus specimens were analyzed for the expression of human Cats V, L, and S by Western blot analysis (Figure 5a). The monoclonal antibodies against human Cat V, as well as mAb33/2, which recognizes both human Cats $\mathrm{V}$ and $\mathrm{L}$, revealed the expression of Cat $\mathrm{V}$ in thymus. The $\mathrm{mAb33} / 1$ and a polyclonal antibody against Cat S clearly demonstrated the presence of Cats L and S. All three enzymes were present as mature proteases in the lysosomal preparations. DCG-04, an E-64 derivative containing tyrosyl and biotinyl moieties for detection, allowed the labeling of active cathepsins in thymic lysosomal preparations (Figure 5c). Because human Cats S and V have identical electrophoretic migration properties, we used the highly potent Cat $S$ inhibitor, LHVS, to discriminate between

\section{Figure 5}

Active form of Cat $V$ is expressed in human thymus. (a) Identification of human Cats V, L, and S in human thymus extracts. Equal amounts $(7.5 \mu \mathrm{g})$ of lysosomal fractions from human thymus were subjected to SDS-PAGE ( $10 \%$ gel), followed by Western blot analysis using antihuman Cat $\mathrm{V}$ antibody, antihuman Cat $\mathrm{S}$ antibody (1:2000 dilution), and $\mathrm{mAb} 33 / 1$ and $\mathrm{mAb} 33 / 2$ recognizing human Cat $L$ and human Cats $L$ and V, respectively. (b) Inhibition of recombinant human Cat $\mathrm{S}$ using LHVS inhibitor. Cats $V$ and $\mathrm{S}$ $(10 \mathrm{ng})$ were incubated with $1 \mathrm{nM}$ LHVS at $37^{\circ} \mathrm{C}$ for the indicated time before labeling with DCG-04 as described in Methods. Activity of Cat $S$ was completely blocked by incubation with $1 \mathrm{nM}$ LHVS at $37^{\circ} \mathrm{C}$ for 20 minutes, but that of Cat $V$ was not. Recombinant human Cat $\mathrm{V}$ is present in two isoforms distinguished by differential glycosylation as described in 14. (c) Selective inhibition of Cat $\mathrm{S}$ but not of Cat $\mathrm{V}$ in thymus extracts. Lysosomal fractions $(1.5 \mu \mathrm{g})$ from human thymus were incubated with or without LHVS $(1 \mathrm{nM}$ or $1 \mu \mathrm{M})$ at $37^{\circ} \mathrm{C}$ for 20 minutes before labeling with DCG-04. At $1 \mathrm{nM}$ LHVS concentration, the remaining band represents Cat V, whereas at $1 \mu \mathrm{M}$ LHVS all cathepsins are inhibited and thus not labeled by DCG-04. both enzymes. At an LHVS concentration of $1 \mathrm{nM}$, amounts of recombinant Cat $S$ comparable to the amounts found in thymus are completely inhibited by this inhibitor and thus do not allow the subsequent labeling with DCG-04. In contrast, equal amounts of Cat $\mathrm{V}$ are not inhibited under identical conditions (Figure 5b). Thus, we conclude that the appropriate DCG-04-labeled band in Figure 5c represents active Cat V. On the other hand, $1 \mu \mathrm{M}$ of LHVS completely eliminates the labeling of proteins with DCG-04 demonstrating that the labeled bands constitute, indeed, cathepsin polypeptides.

$C$ at $V$ degrades $I i$, generates CLIP, and allows peptide loading. Recombinant human Cats V, L, and S were used to investigate the degradation of $\mathrm{Ii}$ and the generation of CLIP. The concentration of the cathepsins was determined by active site titration by using E-64. After a 2-hour incubation of purified $\left[{ }^{35} \mathrm{~S}\right]$ methionine-labeled $\alpha \beta \mathrm{Ii}$ complexes with individual cathepsins, all three enzymes revealed their potential to degrade Ii and generate CLIP (Figure $6 a)$. The processing potencies of Cats $S$ and $V$ were very similar and both enzymes revealed a high selectivity for the degradation of Ii, so that even at high enzyme concentrations, the $\alpha$ and $\beta$ chains remained intact. In contrast, recombinant human Cat L was significantly less selective. Under the conditions used in the experiment, complete degradation of Ii and the release of CLIP already occurred at a concentration of $5 \mathrm{nM}$ Cat $\mathrm{L}$, whereas for Cats $\mathrm{S}$ and $\mathrm{V}$ enzyme concentrations of $50 \mathrm{nM}$ were required. At concentrations higher than $10 \mathrm{nM}$, Cat $\mathrm{L}$ destroyed the MHC class II complex, including CLIP, indicating a nonspecific activity of Cat $\mathrm{L}$ at higher enzyme concentrations.

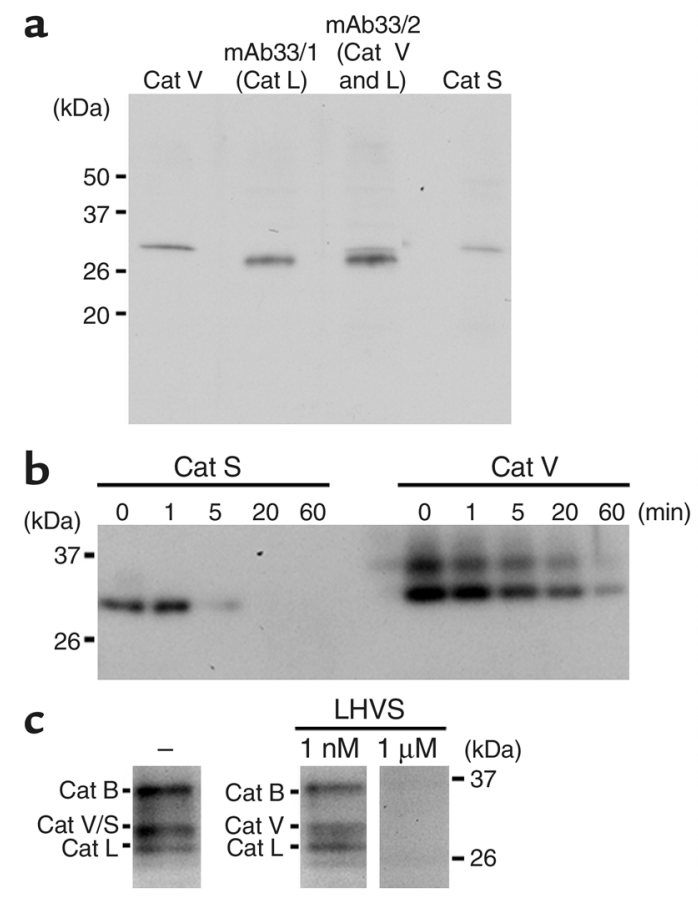




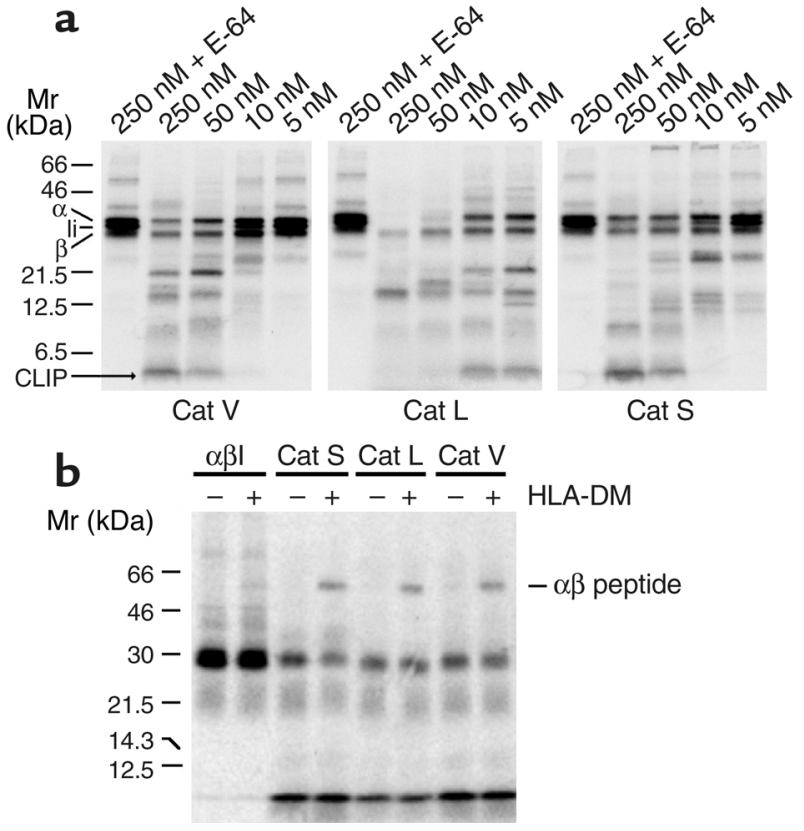

Next, we investigated whether Cat-processed MHC class II complexes were able to bind an antigenic peptide. Radiolabeled DR3- $\alpha \beta$ Ii complexes were digested with Cats V $(50 \mathrm{nM})$, L $(10 \mathrm{nM})$, or S $(50 \mathrm{nM})$. After inactivation of the Cat activities by E-64, the DR3-specific MOMP peptide was incubated with the samples in the presence and absence of HLA-DM for 2 hours at $37^{\circ} \mathrm{C}$. Separation by SDS-PAGE revealed the loading of MOMP peptide on $\alpha \beta$ complexes processed by Cats V, L, and S (Figure 6b). This result clearly indicates that Cats V, S, and $\mathrm{L}$ are capable of processing $\alpha \beta$ Ii into a biologically active complex that has the capability to exchange CLIP for antigenic peptides in the presence of HLA-DM.

Overexpression of Cat $V$ in thymi of $M G$ patients. An autoimmune disease most frequently associated with thymic pathology in humans is MG, and a defect in $T$ cell selection has been frequently suggested as an underlying cause for this disease. Because our data strongly suggest that Cat $\mathrm{V}$ controls the conversion of Ii into CLIP and regulates the class II antigen presentation machinery in TECs, we decided to examine the expression levels of Cat $\mathrm{V}$ in the thymus from MG patients. Hence, the relative gene expression of Cat $\mathrm{V}$ was compared in thymi from normal healthy controls and in MG patients (Figure 7a). Strikingly, thymi from patients showed an overexpression of this protease, both in cases with thymitis and with thymoma, when compared with normal thymus $(P<0.05$, two-tailed Student $t$ test). Expression levels of Cats L and S, as well as other proteases such as Cats B and D and a recently described thymus-specific serine protease were not significantly different in patients and healthy controls (data not shown). Interestingly, samples from non-MG-associated thymomas (26) that were selected for its low-thymocyte contents showed no difference

\section{Figure 6}

Degradation of li by human Cats V, L, and S and peptide loading on cathepsin-processed $\alpha \beta$ li complexes. (a) DA6.147 affinity column purified $\left[{ }^{35} \mathrm{~S}\right.$ ] methionine-labeled HLA-DR3 $\alpha \beta$ li complexes were digested with purified recombinant human Cats $V, L$, and $S$ at the indicated concentrations in sodium acetate buffer, $\mathrm{pH} 5.0$ as described in the Methods section. The digested products were separated by SDS-PAGE using $15 \%$ acrylamide gels. E- 64 inactivated cathepsins at the $250 \mathrm{nM}$ concentration were used as controls. All three cathepsins were capable to degrade li and to generate CLIP. Cats $V$ and $S$ were specific for the processing of li at enzyme concentrations expected to be present in the endosomallysosomal compartment, whereas Cat L activity was nonspecific at concentrations higher than $10 \mathrm{nM}$. The positions of the individual class II $\alpha$ and $\beta$ chains, the li chain, CLIP, and the $\alpha \beta$ peptide dimers are indicated on each gel. (b) Affinity purified radiolabeled HLA-DR $\alpha \beta$ li complexes were digested with Cat V $(50 \mathrm{nM})$, Cat L $(10 \mathrm{nM})$, and Cat $\mathrm{S}(50 \mathrm{nM})$. The digestion mixtures were inactivated by E-64 and subsequently incubated with the DR3-specific MOMP peptide in the presence or absence of HLA-DM for 2 hours at $37^{\circ} \mathrm{C}$. The formation of $\alpha \beta$ peptides is shown after SDS-PAGE using $11.25 \%$ acrylamide gels.

in the expression of Cat $\mathrm{V}$ compared with healthy thymus. We had the chance to compare Cat $\mathrm{V}$ expression in primary cultures of TECs from one MG thymoma patient to TECs from several healthy controls and found it to be about sixfold higher (data not shown), suggesting an increase in Cat $\mathrm{V}$ expression in individual cells rather than an increase in the number of cells that express Cat V. When we looked at protein expression detected by immunohistochemistry on paraffin sections, the thymi from MG patients revealed the same pattern for Cats V, L, and S expressions as we had seen in normal donors. However, $\mathrm{Cat} \mathrm{V}$ in the cortex of MG thymi appeared clearly upregulated when compared with normal thymi. Illustrations $\mathrm{c}$ and $\mathrm{d}$ in Figure 7 show the increased staining of $\mathrm{mAb} 33 / 2$ in the cortex of a thymus from a patient with MG thymitis compared with illustration to a normal thymus (Figure $7 \mathrm{~b}$ ). The medulla did not show any overstaining. The formation of germinal centers inside the thymic medulla is a hallmark of MG thymitis. The light zone of these germinal centers is rich in Cat L-positive cells, and is stained identically by $\mathrm{mAb33} / 1$ and $\mathrm{mAb33} / 2$ (Figure 7, $c$ and $\mathrm{j}$ ), confirming Cat $\mathrm{V}$ as the molecule identified by $\mathrm{mAb} 33 / 2$ in the cortex. In thymoma patients, the overexpression of Cat V in cortex appeared clustered (not shown), suggesting that these are areas of neoplastic transformation in thymi affected by thymoma. In line with the RNA data, we could not observe an increase in Cat L (Figure 7, $\mathrm{g}$ and $\mathrm{h}$, compared with $\mathrm{f}$ ) and Cat $\mathrm{S}$ (not shown) expression in the cortex of MG-affected thymi.

\section{Discussion}

Invariant chain degradation is a critical step in MHC class II maturation for antigen presentation. It has been shown that different APCs use different cysteine 
proteases for this process in the thymus and in the periphery. Cat L is necessary for Ii degradation in mouse cortical epithelial cells (13), whereas Cat $S$ is essential for antigen presentation in peripheral APCs (10). The differential expression of cathepsins in functionally distinct APCs opens the possibility for a selective therapeutic targeting of thymic versus peripheral antigen presentation. This highlights the necessity of an unambiguous identification of the proteases involved. We have recently described human Cat $\mathrm{V}$ as a novel member of the papain family (14). Although this protease shares a protein sequence identity as high as $80 \%$ with human Cat $\mathrm{L}$, its tissue distribution is strikingly different from that of Cat L. Although human Cat $L$ was found in most tissues, the expression of Cat $\mathrm{V}$ was restricted to thymus and testis. Using quantitative real-time PCR, we have analyzed the mRNA expression profiles of human Cats V, L, and $S$ in normal thymus and PBMCs. In agreement with previous reports, we found that Cat $\mathrm{S}$ was expressed at much higher levels in peripheral blood monocytes, whereas Cat $\mathrm{L}$ revealed similar expression levels in thymus and PBMCs and Cat V was found only in thymus. Moreover, absolute quantification of thymic mRNA expression showed that Cat $\mathrm{V}$ is expressed at 75-fold higher levels than that of Cat $\mathrm{L}$ (data not shown). Furthermore, the expression of Cat $V$ was restricted to TECs, indicating that in this compartment of the human thymus, Cat V, but not Cat L, is the predominant cysteine protease. Using monoclonal antibodies that allowed discriminating between Cats $\mathrm{V}$ and $\mathrm{L}$ expression, we confirmed the selective expression of Cat $\mathrm{V}$ in cortical thymic stroma. Cat $\mathrm{L}$ expression was restricted to vesiculated medullary macrophage-like cells and a few cortical mononuclear cells. It should be noted that the antibodies used do not permit the exclusion of Cat $\mathrm{V}$ in these macrophage-like cells.

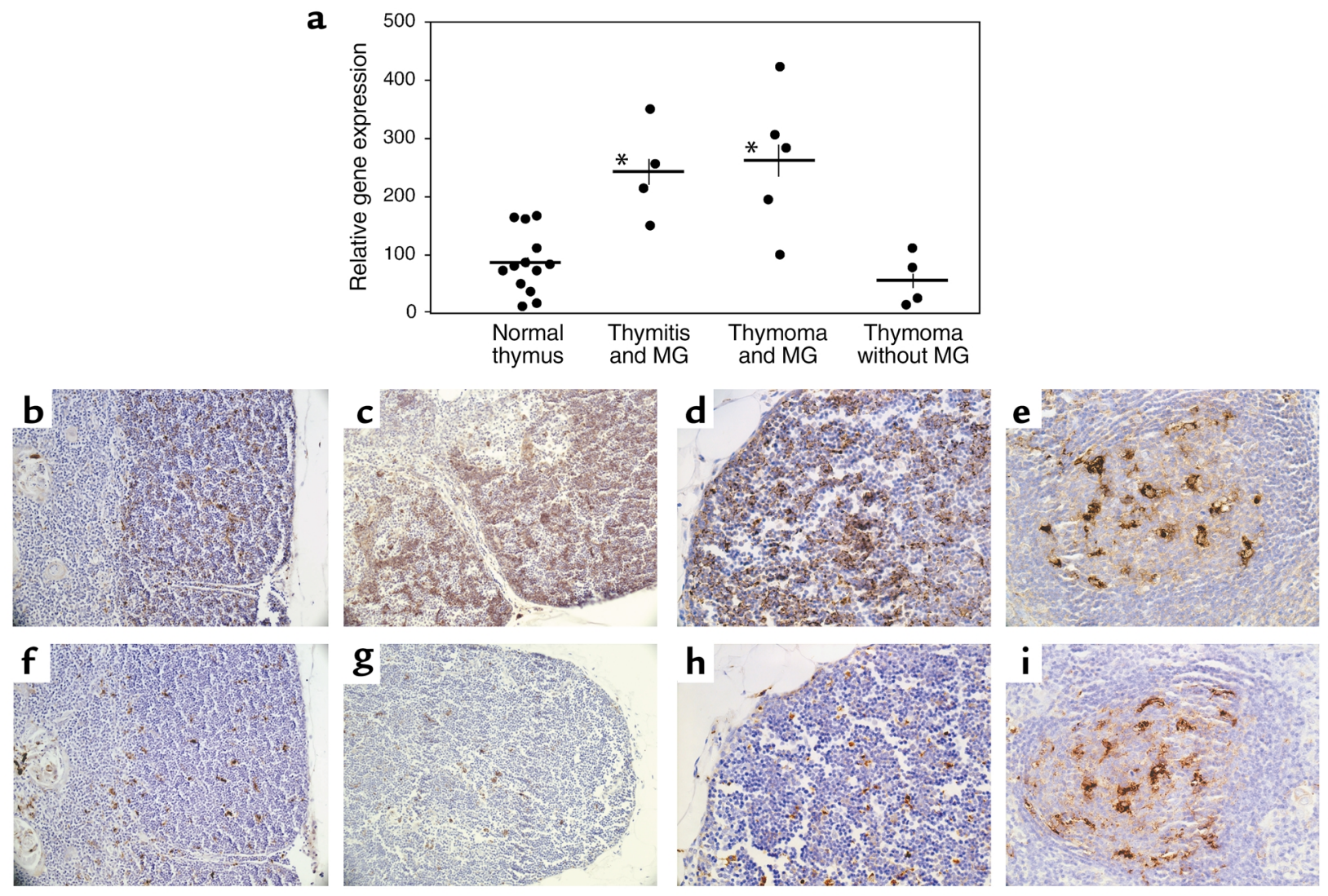

\section{Figure 7}

Overexpression of Cat $\mathrm{V}$ in the thymus of patients with MG. (a) mRNA was extracted from representative blocks of normal or pathologic thymi and analyzed by real-time RT-PCR. Cat $V$ is overexpressed in the thymus of patients with MG, both thymoma $(P=0.006)$ and thymitis $(P=0.004)$ compared with healthy controls or with thymoma patients without myasthenia. ${ }^{*} P<0.001$. (b-i) Mouse monoclonal antihuman Cat $L$ antibodies $m A b 33 / 2$ (cross reacts with Cats $V$ and $L, \mathbf{b}-\mathbf{e}$ ) and $m A b 33 / 1$ (f-i, specific for Cat $L$ ) were used to identify Cats $L$ and $V$ in thymi from patients with MG. Panels $\mathbf{b}$ and $\mathbf{f}$ show the staining pattern of $m A b 33 / 2$ (Cats $V$ and $L)$ and $m A b 33 / 1$ (Cat L) respectively in the cortex of sequential sections from normal human thymus. Increased staining for mAb33/2 but not for mAb33/1 is observed in the thymic cortical epithelium of a MG patient with thymitis ( $\mathbf{c}$ and $\mathbf{g}$ ). Detail of cortex at a higher magnification from sequential sections in the thymus of a patient with MG (d and $\mathbf{h})$. Exact pattern of staining with mAbs $33 / 2$ (e) and 33/1 (i) in an area of thymitis within the medulla of the same patient with MG. This area is devoid of thymic epithelial cells, but contains a dense network of Cat Lpositive and thus labeled with both $33 / 1$ and 33/2 mAbs. Magnifications are $\times 200$ in $\mathbf{b}, \mathbf{c}, \mathbf{f}$, and $\mathbf{g}$ and $\times 400$ in $\mathbf{d}, \mathbf{e}, \mathbf{h}$, and $\mathbf{i}$. Tissue sections were counterstained with hematoxylin. 
To demonstrate that Cat $\mathrm{V}$ is enzymatically active in thymus lysates and at the same time to judge the presence of other papain-like cysteine proteases in these samples, the active site-directed labeling with the peptidyl epoxid inhibitor, DCG-04, was used. Because Cats V and $\mathrm{S}$ cannot be separated by SDS-PAGE, we selectively blocked the labeling of Cat $\mathrm{S}$ by preincubating the thymic extract with the potent Cat $S$ inhibitor, LHVS (at $1 \mathrm{nM}$ for 20 minutes) (10). Under these conditions, Cat $\mathrm{V}$ remains labeled. That the labeled bands represent cathepsins was demonstrated by the elimination of all DCG-04 labeling at higher LHVS concentrations. At $1 \mu \mathrm{M}$ inhibitor concentration, LHVS inhibits Cats V, L, S, K, and B. Thus, we suggest, that the DCG-04-labeled band with an apparent molecular mass of $30 \mathrm{kDa}$ in the presence of $1 \mathrm{nM}$ LHVS represents active human Cat V in thymus extracts. Ideally, one would want to analyze lysosomal fractions from pure epithelial cells, which do not contain Cat $S$, but to date, it is technically close to impossible to isolate these cells with enough purity in the amounts necessary for organelle separation. TECs that have been in culture for 1 week are already phenotypically very different from the freshly isolated cells: Cat V expression is reduced, and Cat L starts to appear (our unpublished observations).

Cortical TECs are mainly responsible for positive selection of T cells, whereas B cells, dendritic cells, and medullary epithelial cells seem to be involved in the negative selection process (27). Mechanistic differences of antigen processing and presentation by cortical and medullary TECs have been documented in murine cell lines $(28,29)$. Moreover, distinct profiles of self-peptides were characterized in murine cortical and medullary epithelial cell lines (30), suggesting that a different array of proteases operates in the two compartments. The lack of Cat L expression in the human cortical stroma is in sharp contrast to the reported expression of Cat $\mathrm{L}$ in mouse thymus (13).

Given the fact that Cat $\mathrm{V}$ is expressed mostly in the thymic cortex, the high homology between murine Cat $\mathrm{L}$ and human Cat $\mathrm{V}$, and the results from the Cat $\mathrm{L} \mathrm{KO}$ mice, we propose that, in humans, Cat V has overtaken the role of Cat L in the positive selection of thymocytes. This assumption is supported by our finding that Cat V selectively degrades Ii complexed to MHC and generates a 3-kDa peptide identical to CLIP in size, with similar efficiency to Cat $\mathrm{S}$. The immunologically relevant degradation of Ii by $\mathrm{Cat} V$ is reflected by the efficient loading of antigenic peptide to DR3- $\alpha \beta$ complexes in the presence of HLA-DM (31). In contrast, human Cat L activity revealed no such substrate specificity. Although no significant degradation of the $\alpha \beta$ complex was observed at enzyme concentrations as high as $250 \mathrm{nM}$ for the Cats V and S, Cat L degraded the complex already at $50 \mathrm{nM}$. Although the concentrations of cathepsins in MHC compartments are unknown, concentrations of Cats L and B have been calculated as high as $1 \mathrm{mM}$ in lysosomes (32). At least for Cat L, this would very likely destroy class II complexes rather than leading to productive antigen presentation. Thus, similar to Cats S, L, and F, we have found that $\mathrm{Cat} V$ promotes the degradation of the intermediate product Ii p10 into CLIP.

MG is a prototypic autoimmune disease targeting acetylcholine receptors of skeletal muscle (reviewed in ref. 33). MG is almost invariably associated with thymic pathologies such as thymitis and thymic tumors, suggesting an intrinsic thymic defect in T cell selection as an underlying cause for $\mathrm{MG}(34,35)$. It has been found that among the various types of thymomas, those associated with MG are characterized by prominent thymopoesis (26) and an increased proportion of recent thymic emigrant $T$ cells are found in the periphery of $M G$ patients $(36,37)$. It is thus reasonable to assume that an altered molecular microenvironment in the thymus promotes the maturation of thymocytes (38) including those with an autoimmune potential (39). Increased numbers of thymocytes may bypass the physiologic check points of selection that usually involves interaction with self-peptide-loaded MHC molecules on specialized thymic APC. We have demonstrated a selective overexpression of Cat $\mathrm{V}$ in thymi of MG patients both with thymitis and thymoma when compared with normal controls and nonMG-associated thymomas, both by real-time PCR and immunohistochemical analysis. It must be noted that the non-neoplastic part of a thymoma often shows thymitis. In contrast, expression of other cathepsins was not significantly different between patients and controls. Of note, even samples from non-MG-associated thymomas that were selected for their low thymocyte contents showed no difference in the expression of Cat $\mathrm{V}$ compared with control thymus. Although the immediate effects of higher levels of Cat $\mathrm{V}$ in the cortical epithelial cells are unknown, a possible outcome would be an imbalance of the positive/negative selection mechanisms, leading to a higher number of autoreactive cells in the periphery. In analogy with these data, the Cat S KO mice were found less susceptible to collagen-induced arthritis than their WT littermates (11), and although the mechanism for this resistance is not known, it is plausible to think of different kinetics of antigen presentation in the peripheral APC caused by the lack of Cat S.

In summary, we have demonstrated that Cat $V$ adds to the list of proteases that degrade Ii, and that it is crucial for Ii degradation in human cortical TECs. The overexpression of Cat V in MG-associated thymic abnormalities may allow the chain of events that lead to autoimmune disease.

Note added in proof. While this paper was being reviewed, an article implicating Cat $S$ and not Cat $V$ in the degradation of Ii in the human thymus was published by Bania et al. (40). In their article, however, the data on thymus is restricted to RT-PCR performed on a cell line derived from cortical epithelium (P1.4D6) 
and a thymic tumor, which could explain the differences with the data generated from normal donors in our laboratory.

\section{Acknowledgments}

We would like to thank Alex Marx (Würzburg, Germany) for providing control thymomas; I. Santamaria for the gift of mAb 236-2D8; M. Heinemann for surgical specimens; Simone Pöschel, Petra Schroth, and Verena Henkel for outstanding technical work, and G. Schwarz for preparing the thymic lysosomes. H.-G. Rammensee and J. Dichgans are thanked for their support. This work was supported by the DFG/SFB 510 (A. Melms, E. Tolosa), the European Community, and the Association Francaise contre les Myopathies (A. Melms, E. Tolosa). E. Tolosa was a Marie Curie Postdoctoral Fellow.

1. Riese, R.J., and Chapman, H.A. 2000. Cathepsins and compartmentalization in antigen presentation. Curr. Opin. Immunol. 12:107-113.

2. Villadangos, J.A., and Ploegh, H.L. 2002. Proteolysis in MHC class II antigen presentation: who's in charge? Immunity. 12:233-239.

3. Manoury, B., et al. 1998. An asparaginyl endopeptidase processes a microbial antigen for class II MHC presentation [see comments]. Nature. 396:695-699.

4. Driessen, C., Lennon-Dumenil, A.M., and Ploegh, H.L. 2001. Individual cathepsins degrade immune complexes internalized by antigenpresenting cells via Fcgamma receptors. Eur. J. Immunol. 31:1592-1601.

5. Cresswell, P. 1994. Assembly, transport, and function of MHC class II molecules. Annu. Rev. Immunol. 12:259-293.

6. Malcherek, G., Gnau, V., Jung, G., Rammensee, H.G., and Melms, A. 1995. Supermotifs enable natural invariant chain-derived peptides to interact with many major histocompatibility complex-class II molecules. J. Exp. Med. 181:527-536.

7. Robbins, N.F., Hammond, C., Denzin, L.K., Pan, M., and Cresswell, P. 1996. Trafficking of major histocompatibility complex class II molecules through intracellular compartments containing HLA-DM. Hum. Immunol. 45:13-23.

8. Riese, R.J., et al. 1998. Cathepsin S activity regulates antigen presentation and immunity. J. Clin. Invest. 101:2351-2363.

9. Denzin, L.K., and Cresswell, P. 1995. HLA-DM induces CLIP dissociation from MHC class II alpha beta dimers and facilitates peptide loading. Cell. 82:155-165.

10. Riese, R.J., et al. 1996. Essential role for cathepsin S in MHC class IIassociated invariant chain processing and peptide loading. Immunity. 4:357-366

11. Nakagawa, T.Y., et al. 1999. Impaired invariant chain degradation and antigen presentation and diminished collagen-induced arthritis in cathepsin S null mice. Immunity. 10:207-217.

12. Shi, G.P., et al. 2000. Role for cathepsin F in invariant chain processing and major histocompatibility complex class II peptide loading by macrophages. J. Exp. Med. 191:1177-1186.

13. Nakagawa, T., et al. 1998. Cathepsin L: critical role in Ii degradation and CD4 T cell selection in the thymus. Science. 280:450-453.

14. Bromme, D., Li, Z., Barnes, M., and Mehler, E. 1999. Human cathepsin $V$ functional expression, tissue distribution, electrostatic surface potential, enzymatic characterization, and chromosomal localization. Biochemistry. 38:2377-2385.

15. Santamaria, I., et al. 1998. Cathepsin L2, a novel human cysteine proteinase produced by breast and colorectal carcinomas. Cancer Res. 58:1624-1630.

16. Itoh, R., Kawamoto, S., Adachi, W., Kinoshita, S., and Okubo, K. 1999.
Genomic organization and chromosomal localization of the human cathepsin L2 gene. DNA Res. 6:137-140.

17. Schroter, C.J., et al. 1999. A rapid method to separate endosomes from lysosomal contents using differential centrifugation and hypotonic lysis of lysosomes. J. Immunol. Methods. 227:161-168.

18. Bromme, D., and McGrath, M.E. 1996. High level expression and crystallization of recombinant human cathepsin S. Protein Sci. 5:789-791.

19. Barrett, A.J., et al. 1982. L-trans-Epoxysuccinyl-leucylamido(4-guanidino)butane (E-64) and its analogues as inhibitors of cysteine proteinases including cathepsins B, H and L. Biochem. J. 201:189-198.

20. Weber, E., Bahn, H., and Gunther, D. 1997. Monoclonal antibodies against cathepsin $\mathrm{L}$ and procathepsin $\mathrm{L}$ of different species. Hybridoma. 16:159-166.

21. Nissler, K., et al. 1999. The half-life of human procathepsin S. Eur. J. Biochem. 263:717-725.

22. Greenbaum, D., Medzihradszky, K.F., Burlingame, A., and Bogyo, M. 2000. Epoxide electrophiles as activity-dependent cysteine protease profiling and discovery tools. Chem. Biol. 7:569-581.

23. Machamer, C.E., and Cresswell, P. 1984. Monensin prevents terminal glycosylation of the $\mathrm{N}$ - and O-linked oligosaccharides of the HLA-DRassociated invariant chain and inhibits its dissociation from the alphabeta chain complex. Proc. Natl. Acad. Sci. U. S. A. 81:1287-1291.

24. Riberdy, J.M., and Cresswell, P. 1992. The antigen-processing mutant T2 suggests a role for MHC-linked genes in class II antigen presentation. J. Immunol. 148:2586-2590.

25. Denzin, L.K., Robbins, N.F., Carboy-Newcomb, C., and Cresswell, P. 1994. Assembly and intracellular transport of HLA-DM and correction of the class II antigen-processing defect in T2 cells. Immunity. 1:595-606.

26. Muller-Hermelink, H.K., and Marx, A. 1999. Pathological aspects of malignant and benign thymic disorders. Ann. Med. 31(Suppl. 2):5-14.

27. Laufer, T.M., Glimcher, L.H., and Lo, D. 1999. Using thymus anatomy to dissect T cell repertoire selection. Semin. Immunol. 11:65-70.

28. Kasai, M., Kominami, E., and Mizuochi, T. 1998. The antigen presentation pathway in medullary thymic epithelial cells, but not that in cortical thymic epithelial cells, conforms to the endocytic pathway. Eur.J. Immunol. 28:1867-1876.

29. Oukka, M., et al. 1997. Selectivity of the major histocompatibility complex class II presentation pathway of cortical thymic epithelial cell lines. Eur. J. Immunol. 27:855-859.

30. Kasai, M., Kropshofer, H., Vogt, A.B., Kominami, E., and Mizuochi, T. 2000. CLIP-derived self peptides bound to MHC class II molecules of medullary thymic epithelial cells differ from those of cortical thymic epithelial cells in their diversity, length, and C-terminal processing. Eur. J. Immunol. 30:3542-3551.

31. van Ham, S.M., et al. 1996. Human histocompatibility leukocyte antigen (HLA)-DM edits peptides presented by HLA-DR according to their ligand binding motifs. J. Exp. Med. 184:2019-2024.

32. Xing, R., Addington, A.K., and Mason, R.W. 1998. Quantification of cathepsins B and L in cells. Biochem. J. 332:499-505.

33. Vincent, A., Palace, J., and Hilton-Jones, D. 2001. Myasthenia gravis. Lancet. 357:2122-2128.

34. Bofill, M., et al. 1985. Microenvironments in the normal thymus and the thymus in myasthenia gravis. Am. J. Pathol. 119:462-473.

35. Wekerle, H., and Ketelsen, U.P. 1997. Intrathymic pathogenesis and dual genetic control of myasthenia gravis. Lancet. 1:678-680.

36. Buckley, C., Douek, D., Newsom-Davis, J., Vincent, A., and Willcox, N. 2001. Mature, long-lived CD4+ and CD8+ T cells are generated by the thymoma in myasthenia gravis. Ann. Neurol. 50:64-72.

37. Sempowski, G., et al. 2001. Effect of thymectomy on human peripheral blood T cell pools in myasthenia gravis. J. Immunol. 166:2808-2817.

38. Strobel, P., et al. 2002. Paraneoplastic myasthenia gravis correlates with generation of mature naive $\mathrm{CD} 4(+) \mathrm{T}$ cells in thymomas. Blood. 100:159-166.

39. Melms, A., et al. 1988. Thymus in myasthenia gravis. Isolation of Tlymphocyte lines specific for the nicotinic acetylcholine receptor from thymuses of myasthenic patients. J. Clin. Invest. 81:902-908.

40. Bania, J., et al. 2003. Human cathepsin S, but not cathepsin L, degrades efficiently MHC class II-associated invariant chain in nonprofessional APCs. Proc. Natl. Acad. Sci. U. S. A. 100:6664-6669. 